\title{
Chronic Management of Hypertension after Stroke: The Role of Ambulatory Blood Pressure Monitoring
}

\author{
Luis Castilla-Guerra, ${ }^{\mathrm{a}, \mathrm{b}}$ Maria del Carmen Fernandez-Moreno ${ }^{\mathrm{a}}$ \\ ${ }^{a}$ Department of Neurology, Hospital de Valme, University of Seville, 41014 Seville, Spain \\ ${ }^{b}$ Department of Internal Medicine, Hospital Universitario Virgen Macarena, 41071 Seville, Spain
}

Hypertension is the most important potentially reversible risk factor for stroke in all age groups; high blood pressure (BP) is also associated with increased risk of recurrent stroke in patients who have already had an ischemic or hemorrhagic event. Twenty-four hour ambulatory BP monitoring (ABPM) has become an important tool for improving the diagnosis and management of hypertension, and is increasingly used to assess patients with hypertension. Nevertheless, although ABPM devices are increasingly used for assessment of hypertension, their value in the chronic management of hypertension in patients with stroke has not been systematically studied. In fact, among large-scale randomized trials for secondary stroke prevention, only the Morbidity and Mortality After Stroke, Eprosartan Compared With Nitrendipine for Secondary Prevention trial included 24-hour ABPM. ABPM has demonstrated chronic disruption of the circadian rhythm of BP after acute phase of stroke and has shown higher sensitivity compared to office BP in evaluating the effectiveness of antihypertensive treatment among stroke survivors. High 24-hour BP is an independent predictor for cerebrovascular events, brain microbleeds, and subsequent development of dementia. Nevertheless, although stroke care guidelines endorse the importance of hypertension management, the specific role of ABPM among stroke survivors after the acute phase of disease has not been established. Further studies are needed to clarify whether routine application of ABPM among these patients should be recommended.

Keywords Ambulatory blood pressure monitoring; Blood pressure; Hypertension; Stroke; Secondary prevention

\author{
Correspondence: Luis Castilla-Guerra \\ Department of Neurology, Hospital de \\ Valme, University of Seville, 41014 \\ Seville, Spain \\ Tel: +44-955077261 \\ Fax: +44-955077260 \\ E-mail: castillafernandez@hotmail.com
}

Received: March 26, 2015

Revised: November 12, 2015

Accepted: November 13, 2015

The authors have no financial conflicts of interest.

\section{Introduction}

High blood pressure (BP) is the most important potentially reversible risk factor for stroke in all age groups, with a continuous association between both systolic and diastolic BPs (SBPs and DBPs, respectively) and risk of stroke, particularly for intracerebral hemorrhage. ${ }^{1} \mathrm{SBP}>115 \mathrm{mmHg}$ explains $60 \%$ of the population-attributable risk of stroke. ${ }^{2}$ High BP is also associated with increased risk of recurrent stroke in patients who have already had an ischemic or hemorrhagic event. ${ }^{3}$

A recent meta-analysis of 16 randomized controlled trials and 40,292 patients with history of stroke (ischemic, transient ischemic attack [TIA], or hemorrhagic), with and without hypertension, demonstrated that BP-lowering treatment reduced the risk of recurrent stroke (relative risk reduction, 18\%; 95\% confidence interval [CI], 9\%-26\%). ${ }^{4}$ The meta-regression analysis also revealed that each $10-\mathrm{mmHg}$ reduction in SBP is associated with 33\% (95\% CI, 9\%-51\%) reduction 


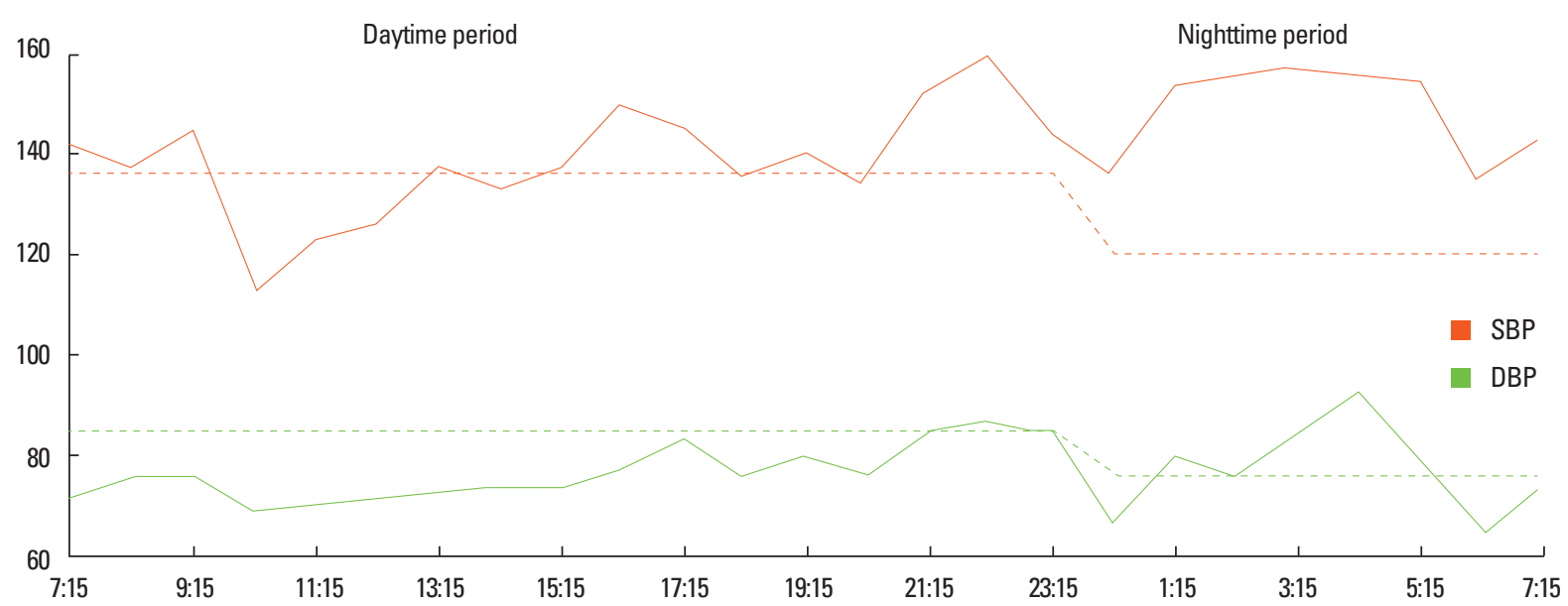

Figure 1. Ambulatory blood pressure monitoring in a patient with recent ischemic stroke, showing a reversed dipper blood pressure pattern. SBP, systemic blood pressure; DBP, diastolic blood pressure.

in the risks of recurrent stroke. ${ }^{4}$

Twenty-four hour ambulatory BP monitoring (ABPM) has become an important tool for improving the diagnosis and management of hypertension, and it is increasingly used to assess patients with hypertension. This trend is supported by evidence that 24-hour BP profiles are superior to isolated clinic BPs in predicting future cardiovascular events and target organ damage. $^{5}$

In fact, recent UK National Institute for Health and Care Excellence guidelines for diagnosis and management of hypertension recommend that routine ABPM to confirm hypertension diagnoses. ${ }^{6}$ As a recent review of international guidelines indicates, these are the first guidelines to clearly state that ABPM should be provided to anyone suspected of having hypertension based on an elevated clinic BP measurement, taking into account new evidence not available at the time of publication of previous guidelines. This change in practice is in contrast to most other countries, where ABPM is currently discouraged by lack of reimbursement; other guidelines do not currently make the same recommendation for ABPM, with the European Society of Hypertension guidelines specifying that ABPM 'should not be regarded as a substitute for information derived from conventional BP measurements', though recommending its use in suspected white-coat and masked hypertension, to identify hypotension and resistant hypertension, and to assess drug efficacy and nocturnal dipping status. ${ }^{78}$

The information provided by 24-hour ABPM includes daytime and nighttime BP profiles, day-night BP differences, morning $\mathrm{BP}$ increase, and BP variability (Figure 1 ). Studies have identified associations between 24-hour ABPM parameters and hypertensive target organ damage, including left ventricular hypertrophy, microalbuminuria, intima media thickness, retinal changes, pulse wave velocity, and silent brain damage. ${ }^{8,9}$

Nevertheless, although ABPM devices are increasingly used for assessment of hypertension, their value in patients after the acute phase of stroke has not been systematically studied. This is an important issue because hypertension is a major cause of stroke recurrence. This paper reviews the role of ABPM in the chronic management of hypertension in patients with stroke.

\section{Evidence from randomized controlled trials on BP management for secondary prevention of stroke}

To date, only a few randomized controlled trial studies have assessed the role of BP management in secondary prevention of stroke (Table 1).

\section{Post-Stroke Antihypertensive Treatment Study (PATS) trial $^{10}$}

The Post-Stroke Antihypertensive Treatment Study trial was a randomized, double-blind and placebo-controlled trial intended to determine whether antihypertensive treatment could reduce the risk of fatal and nonfatal stroke incidence in patients with a history of stroke or TIA. Monotherapy with the diuretic indapamide $(2.5 \mathrm{mg} /$ day $)$ was compared with placebo in 5,665 patients. The findings of this trial indicated that a $\mathrm{BP}$ reduction of $5 / 2 \mathrm{mmHg}$ with indapamide reduced the incidence of fatal and nonfatal stroke by $29 \%$ in patients with a history of stroke or TIA.

\section{Perindopril Protection Against Recurrent Stroke Study (PROGRESS) trial'11}

The Perindopril Protection Against Recurrent Stroke Study 
Table 1. Trials on blood pressure lowering and secondary stroke prevention

\begin{tabular}{|c|c|c|c|c|c|c|c|}
\hline Study & Population & Event & Treatment & Period (yr) & $\mathrm{BP}$ reduction & RR cerebrovascular events & ABPM \\
\hline PATS & 5,665 & $\begin{array}{l}\text { IS, HS, TIA with and } \\
\text { without HT }\end{array}$ & $\begin{array}{l}\text { Indapamide } 2.5 \mathrm{mg} \\
\text { vs. placebo }\end{array}$ & 2 & $5 / 2 \mathrm{mmHg}$ & RR: $29 \%$ & NO \\
\hline \multirow[t]{2}{*}{ PROGRESS } & 6,105 & $\begin{array}{l}\text { IS, HS, TIA, previous } 5 \\
\text { years (mean } 8 \text { months) } \\
\text { with and without HT }\end{array}$ & Perindopril vs. placebo & 3.9 & $9 / 4 \mathrm{mmHg}$ & $\begin{array}{l}\text { RRR: } 28 \% \\
\text { (95\% Cl 17-38) }\end{array}$ & NO \\
\hline & & & $\begin{array}{l}\text { Perindopril plus indapamide } \\
\text { vs. placebo }\end{array}$ & & $\begin{array}{l}\text { Perindopril: } 5 / 3 \mathrm{mmHg} \\
\text { Perindorpil plus indapamide: } \\
12 / 5 \mathrm{mmHg}\end{array}$ & $\begin{array}{l}\text { Perindopril: } 5 \% \\
\text { Perindopril plus } \\
\text { indapamide: } 43 \%\end{array}$ & \\
\hline MOSES & 1,405 & $\begin{array}{l}\text { IS, HS, TIA previous } \\
2 \text { years, hypertensive } \\
\text { patients }\end{array}$ & $\begin{array}{l}\text { Eprosartan } 600 \text { mg } \\
\text { vs. Nitrendipine } 10 \text { mg }\end{array}$ & 2.5 & $\begin{array}{l}\text { Eprosartan group: } \\
13 / 3 \mathrm{mmHg} \\
\text { Nitrendipine group: } \\
16 / 7 \mathrm{mmHg}\end{array}$ & $\begin{array}{l}\text { IDR of } 0.75 \\
\text { (95\% Cl 0.58-0.97) }\end{array}$ & YES \\
\hline PRoFESS & 20,332 & $\begin{array}{l}\text { IS previous } 3 \text { months } \\
\text { (mean } 15 \text { days) with } \\
\text { and without HT }\end{array}$ & $\begin{array}{l}\text { Termisaltan } 80 \mathrm{mg} \\
\text { vs. Placebo }\end{array}$ & 2.5 & $\begin{array}{l}3.8 / 2.0 \mathrm{mmHg} \text { lower than } \\
\text { placebo }\end{array}$ & $\begin{array}{l}\text { HR: } 0.95 \\
\qquad(95 \% \mathrm{Cl} 0.86-1.04 ; P=0.23)\end{array}$ & NO \\
\hline SPS3 & 3,020 & $\begin{array}{l}\text { MRI-defined symptomatic } \\
\text { lacunar infarctions in the } \\
\text { previous } 180 \text { days }\end{array}$ & $\begin{array}{l}\text { Antihypertensives } \\
\text { prescribed by the local } \\
\text { study physician }\end{array}$ & 3.7 & $\begin{array}{l}\text { Systolic-blood-pressure } \\
\text { target of } 130-149 \mathrm{mmHg} \\
\text { or less than } 130 \mathrm{mmHg}\end{array}$ & $\begin{array}{l}\text { HR } 0.81 \\
\qquad(95 \% \mathrm{Cl} 0.64-1.03, P=0.08)\end{array}$ & NO \\
\hline
\end{tabular}

BP, Blood Pressure; RR, Risk Reduction; ABPM, Ambulatory blood Pressure Monitoring; IS, Ischemic stroke; HS, Hemorrhagic Stroke; TIA, Transient ischemic attack; RRR, relative risk reduction; HT, Hypertension; IDR, incidence density ratio; MRI, magnetic resonance image.

randomized 6,105 patients with history of ischemic stroke or TIA with and without hypertension to three groups that received perindopril ( $4 \mathrm{mg} /$ day) alone, in combination with indapamide $(2.5 \mathrm{mg} /$ day $)$, or placebo.

After a mean follow up of 3.9 years, the active treatment group as a whole had significantly fewer strokes and major vascular events. Treatment reduced BP an average of 9 and 4 $\mathrm{mmHg}$ SBP and DBP, respectively, and reduced the relative risk of stroke by $28 \%$ ( $95 \%$ CI, 17-38) versus placebo. However, a pre-specified subgroup analysis showed that, although participants treated with the combination of perindopril plus indapamide had average SBP and DBP decreases of 12 and $5 \mathrm{mmHg}$ and significantly lower stroke risk than patients who received double placebo ( $43 \%$ risk reduction; $95 \%$ CI $30 \%$ to $45 \%$ ), patients treated with perindopril alone showed a BP reduction of $5 / 3 \mathrm{mmHg}$ and had a risk of stroke that was not discernibly different from placebo ( $5 \%$ risk reduction, $-19 \%$ to $23 \%$ ).

\section{Morbidity and Mortality After Stroke, Eprosartan Compared with Nitrendipine for Secondary Prevention (MOSES) trial' ${ }^{12}$}

The Morbidity and Mortality After Stroke, Eprosartan Compared with Nitrendipine for Secondary Prevention trial was the first to compare the relative benefit of particular antihypertensive drugs for secondary prevention of stroke. It compared the efficacy of treatment with eprosartan (an angiotensin II type receptor blocker, $600 \mathrm{mg} /$ day) and nitrendipine (a calcium channel blocker) in 1,045 hypertensive patients who had had a cerebral event in the previous 24 months. BP reductions were similar with the two agents. Treatment with eprosartan resulted in a $25 \%$ stroke risk reduction compared to nitrendipine (236 events; incidence density ratio 0.75 ; $95 \%$ CI: 0.58 to $0.97)$; there was also a reduction in the risk of primary composite events (death, or cardiovascular or cerebrovascular events; incidence density ratio $0.79 ; 95 \% \mathrm{CI}, 0.66$ to 0.96 ). A reduction in TIAs accounted for most of the benefit in cerebrovascular events, with no significant difference in ischemic strokes, and a more traditional analysis of the time to first cerebrovascular event did not show a benefit of eprosartan. However, the study had several methodological limitations, including its open design.

\section{Prevention Regimen for Effectively Avoiding Second Strokes (PRoFESS) trial $^{13}$}

The Prevention Regimen for Effectively Avoiding Second Strokes assessed 20,332 patients with recent ischemic stroke (within 90 days of the event) randomized to either angiotensin II type receptor blocker, $(80 \mathrm{mg} /$ day telmisartan, $\mathrm{n}=10,146)$, or placebo $(n=10,186)$ on a background of standard antihypertensive therapy as part of a $2 \times 2$ factorial design study, which also included aspirin plus extended-release dipyridamole versus clopidogrel. Telmisartan was not associated with a reduction in recurrent stroke (hazard ratio [HR], 0.95; 95\% CI: 0.86 to 1.04$)$ or major cardiovascular events (HR, 0.94; 95\% CI: 0.87 to 1.01 ) during a mean 2.5-year follow-up period. The BP-lowering arm in the trial was statistically underpowered. Nonadherence to telmisartan and more aggressive treatment with other antihypertensive medications in the pla- 
cebo group reduced the difference in $\mathrm{BP}$ between the treatment groups (SBP differed by $5.4 \mathrm{mmHg}$ at 1 month and 4.0 $\mathrm{mmHg}$ at one year) and may have reduced the impact of treatment on stroke recurrence.

\section{Stroke Prevention of Small Subcortical Strokes (SPS3) trial ${ }^{14}$}

The Stroke Prevention of Small Subcortical Strokes trial included 3,020 patients with recent magnetic resonance imagingdefined symptomatic lacunar infarctions divided into two target groups of BP control. Outcomes were compared between those assigned to the "lower target" SBP (below $130 \mathrm{mmHg}$ ) and those treated to achieve a "higher target" (between 130 and $149 \mathrm{mmHg}$ ). The primary endpoint was reduction in all strokes (including ischemic strokes and intracranial hemorrhages). After an average follow-up of about 3.7 years, non-significant rate reductions were seen for all stroke (HR 0.81, 95\% CI: 0.64-1.03, $P=0.08$ ), disabling or fatal stroke (HR 0.81, 95\% CI: $0.53-1.23, P=0.32$ ), and the composite outcome of myocardial infarction or vascular death (HR 0.84, 95\% CI: $0.68-1.04, P=0.32$ ) with the lower target. In addition, the rate of intracerebral hemorrhage was significantly reduced (HR 95\% CI: 0.37, 0.15-0.95, $P=0.03$ ).

Nevertheless, among these randomized controlled trials, only the Morbidity and Mortality After Stroke, Eprosartan Compared With Nitrendipine for Secondary Prevention trial included 24-hour ABPM. The most important goal that trial was to achieve comparable normalization of $\mathrm{BP}$ in both treatment groups. Thus, clinic BP results were confirmed by ABPM in order to exclude differences in BP during 24 hours. Twentyfour-hour ABPM was scheduled for 12-, 24-, and 48-month. Finally, BP was reduced to a comparable extent without significant differences between the two groups during the whole study period. At the end of the study, or at the final visit, the mean values of systolic and DBPs were normalized (eprosartan group 133.2/80.4 mmHg; nitrendipine group 132.7/80.2
$\mathrm{mmHg}$ ). No significant differences between groups were found by 24-hour ABPM. ${ }^{12}$

\section{Evidence from other studies on ABPM for secondary prevention of stroke}

As previously discussed, ABPM is currently considered the best predictor of target organ involvement; thus, accurate definitions of its changes over the 24-hour cycle are of crucial importance in this population.

Many studies have focused on the nature and extent of alterations of circadian BP patterns after acute stroke, and a pathologically reduced or abolished circadian BP variation has been described in acute stroke. ${ }^{15}$ However, studies on alterations of circadian BP patterns after acute phase of stroke are scarce.

Castilla-Guerra et al. ${ }^{16}$ (Table 2) reported a chronic disruption of circadian rhythm of BP after the acute phase of stroke in a prospective study of 101 patients admitted within 24 hours after stroke onset and followed for one year; although the BP tended to decline and normalize weeks or months after stroke with the effect of medication, this abnormal pattern of circadian rhythm persisted in the majority (approximately threequarters) of stroke patients after a one-year follow-up period. The authors found that the normal diurnal variation in BP was abolished in $87.1 \%$ of patients during the acute phase of stroke, in $76.9 \%$ after six months, and in $74.6 \%$ after one year.

In accordance with these results, Sasaki et al. ${ }^{17}$ found that the nocturnal BP reduction was blunted in most $(\sim 90 \%)$ of $48 \mathrm{el}-$ derly bedridden hypertensive patients assessed within 1-3 months after stroke. ABPM has also demonstrated a higher sensitivity compared to office BP for evaluation of the effectiveness of antihypertensive treatment among stroke survivors.

Cugini et al. ${ }^{18}$ found a highly significant proportion of TIA patients (90\%) whose hypertension was not well controlled in a series of 51 patients with a history of recent TIA syndrome and 225 clinically healthy control subjects who under-

Table 2. Main studies with ABPM for secondary prevention of stroke

\begin{tabular}{|c|c|c|c|}
\hline Author & Population & Main findings & Reference \\
\hline Castilla-Guerra et al. & 101 stroke survivors & Chronic disruption of circadian BP rhythm. One-year follow-up period & 16 \\
\hline Sasaki et al. & 48 elderly bedridden hypertensive stroke patients & Nocturnal BP fall was blunted in most ( $90 \%)$ of the patients & 17 \\
\hline Cugini et al. & 51 patients with TIA and 225 control subjects & $\begin{array}{l}\text { Higher sensitivity of ABPM compared to office BP in evaluating the } \\
\text { effectiveness of antihypertensive treatment }\end{array}$ & 18 \\
\hline Zakopoulos et al. & 187 hypertensive stroke survivors & Less effective BP control using ABPM compared to office recordings & 19 \\
\hline Castilla-Guerra et al. & 50 ischemic stroke survivors & High frequency of nocturnal hypotension episodes & 25 \\
\hline Yamamoto et al. & 105 lacunar infarcts & Lack of J-curve & 27 \\
\hline Yamamoto et al. & 177 lacunar strokes & $\begin{array}{l}\text { High 24-hour SBP is an independent predictors for vascular events } \\
\text { and dementia }\end{array}$ & 28 \\
\hline Staals et al. & 123 lacunar strokes & Association with the presence and number of brain microbleeds & 29 \\
\hline Yamamoto et al. & 224 lacunar infarctions & Association with cognitive impairment & 30 \\
\hline
\end{tabular}


went ABPM. In study of 187 first-ever consecutive hypertensive stroke survivors with $\mathrm{ABPM}$, Zakopoulos et al. ${ }^{19}$ later showed effective BP control significantly fewer patients using ABPM (32.1\%) than those using office recordings (43.3\%, $P<0.001$ ), whereas a masked lack of per-treatment blood pressure control (elevated $\mathrm{ABP}$ in the presence of normal office BP levels) was identified in $16 \%$ of the study population.

Similarly, Castilla-Guerra et al. ${ }^{16}$ found conventional clinical recordings to be an unreliable method of control in their prospective study including 88 ischemic and 13 hemorrhagic strokes followed for one year, concluding that ABPM should be routinely performed in this population.

Therefore, the high incidence of inadequately treated patients with a history of recent stroke or TIA confirms that BP should be controlled by ABPM in this population. The results of these studies also suggest that chronomodulation of antihypertensive treatment might be more effective for management of $\mathrm{BP}$ in these patients for secondary prevention of cerebrovascular damage.

\section{$A B P M$ and the $\mathrm{J}$ curve after stroke}

Another important issue is the target $\mathrm{BP}$ in patients after the acute phase of stroke. Currently, the relationship between post-stroke $\mathrm{BP}$ and stroke recurrence remains undetermined, and the optimal control of BP has not been established.

Although the target BP goals or reduction from pretreatment baseline are uncertain and should be individualized, SBP $<140 \mathrm{mmHg}$ and a DBP $<90 \mathrm{mmHg}$ (Class IIa; Level of Evidence B) are typically recommended in daily clinical practice of secondary stroke prevention. ${ }^{20}$

However, these BP figures may be too strict for subjects with recent or subacute stroke and, may lead to hypotension, with the resulting additional risk to these patients. In fact, recent years have seen renewed debate regarding the J-curve in BP after stroke. Several studies have suggested that an excessive lowering of BP can cause ischemic stroke and decline in cognitive function. ${ }^{21}$ These studies also suggest that decreased BP might reduce cerebral blood flow, especially in chronic stroke patients in whom an upward shift of the autoregulatory range was recognized after the acute period had passed.

Two decades ago, Irie and colleagues ${ }^{22}$ reported the J-curve phenomenon to be related to ischemic stroke recurrence. They analyzed 368 stroke patients with a history of hypertension (mean age, 62 years) admitted within three months after stroke onset and observed for at least six months. They found that stroke recurrence rate had a J-curve relative to post-stroke DBP. The stroke recurrence rate was $3.8 \%$ per patient-year among 94 patients with post-stroke DBP of 80 to $84 \mathrm{mmHg}$, significantly lower than the rates of $9.2 \%$ per patient-year $(P<0.05)$ and $11.4 \%$ per patient-year $(P<0.01)$ in those with lower and higher post-stroke diastolic BPs, respectively.

A recent post hoc observational analysis of the Prevention Regimen for Effectively Avoiding Second Strokes trial, which included 20,330 patients with recent ischemic stroke, suggested that SBP maintained in a low-normal range $(<120 \mathrm{mmHg})$ may be associated with increased risk of recurrent stroke, especially within the first six months after the first stroke. ${ }^{23} \mathrm{Sim}$ ilarly, an analysis of the dataset from the Vitamin Intervention for Stroke Prevention (VISP) study involving 3,680 patients with recent noncardioembolic ischemic stroke found an increased risk of recurrent stroke in patients with low-normal SBP levels ( $\mathrm{SBP}<120 \mathrm{mmHg}$ ), especially within the first six months after the first stroke. ${ }^{24}$

It is likely that sustained SBP $<120 \mathrm{mmHg}$ could provoke periods of low BP and compromise cerebral flow. CastillaGuerra et al..$^{25}$ assessed ABPM in stroke survivors and demonstrated that episodes of nocturnal hypotension were very common in this population. In a study using ABPM that included 50 consecutive patients presenting with ischemic stroke in the previous six months, episodes of nocturnal hypotension were observed in 21 patients (42\%), demonstrating that episodes of nocturnal hypotension are very common in patients with recent stroke, very possibly much more than a priori, and based on traditional clinical or office BP measurements. The high frequency of nocturnal hypotension episodes observed in the study suggests that significant proportion of patients is inadvertently subjected to a significant risk of critical reduction in cerebral perfusion. This could be especially important in subjects who have suffered a stroke, in which cerebral autoregulation, responsible for maintaining cerebral blood flow constant over a wide range of $\mathrm{BP}$, is often dysfunctional, causing cerebral perfusion to become more dependent on BP values. ${ }^{26}$

However, Yamamoto et al. ${ }^{27}$ did not observe the J-curve phenomenon or an excessive fall in the nighttime BP leading to cerebral ischemic lesions in a prospective study of 105 patients with symptomatic lacunar infarcts who underwent repeated magnetic resonance imaging and 24-hour BP monitoring with an average follow-up period of 3.2 years in order to elucidate the appropriate BP control level for prevention of silent and symptomatic cerebral infarction. Nevertheless, the authors pointed out that the fact that patient BP was controlled at relatively high levels in the study, and that they restricted their subjects to patients with lacunar infarcts may have contributed to the lack of a J-curve. 


\section{Prognosis value of ABPM after stroke}

As previously discussed, since the introduction of ABPM devices, target organ damage and cardiovascular morbidity and mortality are reportedly more closely correlated with $\mathrm{ABP}$ than casual BP. Moreover, a nondipping status, that is, a state whereby patients exhibit either an absence or reduced nocturnal BP dip, reportedly correlates with more advanced target organ damage and worsened prognosis than patients who maintained a dipping status. Therefore, $\mathrm{ABP}$ values are of prognostic value for the prediction of subsequent morbid events in patients with essential hypertension. ${ }^{4,6}$ However, the prognostic value of ABPM for established cerebrovascular disease has only occasionally been investigated.

In a study of one 177 patients with lacunar strokes and an ABPM performed from 14 to 30 days after stroke without administration of antihypertensive agents and tracked for a mean of 8.9 years of follow-up, Yamamoto et al. ${ }^{28}$ showed that high 24-hour SBP was an independent predictor for vascular events (>145 mmHg vs. < $130 \mathrm{mmHg}$; risk reduction: 10.3; 95\% CI: 1.3 to 81.3 ), and nondipping status (patients who did not exhibit a $>5 \%$ nocturnal dip in SBP) was an independent predictor for subsequent development of dementia (risk reduction: 7.1 ; $95 \%$ CI: 2.2 to 22.0 ).

Among 123 first-ever lacunar stroke patients with a 24-hour ABPM after the acute stroke-phase between one and six months post-stroke), Staals et al. ${ }^{29}$ found 24-hour, day, and night SBP and DBP levels to be significantly associated with the presence and number of brain microbleeds, with odds ratios 1.6 to 2.3 per standard deviation increase in BP. Distinguishing between different locations, various BP characteristics were significantly associated with the presence of deep (or combined deep and lobar) brain microbleeds, but not with purely lobar brain microbleeds.

Finally, other authors ${ }^{30}$ reported that non-dipping status along with extensive small vessel diseases and chronic kidney disease were independently associated with cognitive impairment in a sample of 224 consecutive patients with symptomatic lacunar infarction who underwent magnetic resonance imaging and $A B P M$.

\section{Conclusions}

To prevent recurrence of cerebrovascular disease, adequate control of BP is extremely important. Current evidence suggests the utility of 24-hour ABPM in patients with recent stroke in order to improve rates of $\mathrm{BP}$ control and rational drug treatment.
Nevertheless, although stroke care guidelines endorse the importance of hypertension management, the specific role of ABPM among stroke survivors after the acute phase of disease has not been established. There remain many unsolved questions regarding the use of ABPM after stroke, such as when to perform the test and reasonable thresholds for hypertension diagnosis and treatment based on ABPM after stroke, among others.

Therefore, although introduction of ABPM in clinical practice may improve hypertension treatment after stroke, additional studies are necessary to clarify whether routine application of ABPM among patients with recent stroke should be recommended.

\section{References}

1. Goldstein LB, Adams R, Alberts MJ, Appel LJ, Brass LM, Bushnell CD, et al. Primary prevention of ischemic stroke: a guideline from the American Heart Association/American Stroke Association Stroke Council. Stroke 2006;37:1583-1633.

2. Lawes CM, Vander HS, LawMR, Elliott P, MacMahon S, Rodgers A. Blood pressure and the global burden of disease 2000. Part II: estimates of attributable burden. J Hypertens 2006;24: 423-430

3. Castilla-Guerra L, Fernández-Moreno Mdel C. Update on the management of hypertension for secondary stroke prevention. Eur Neurol 2012;68:1-7.

4. Arima H, Chalmers J. PROGRESS: Prevention of Recurrent Stroke. J Clin Hypertens (Greenwich) 2011;13:693-702.

5. Hansen TW, Kikuya M, Thijs L, Björklund-Bodegård K, Kuznetsova T, Ohkubo T, et al. IDACO Investigators. Prognostic superiority of daytime ambulatory over conventional blood pressure in four populations: a meta-analysis of 7030 individuals. J Hypertens 2007;25:1554-1564.

6. National Institute for Clinical Excellence. Hypertension: management of hypertension in adults in primary care. http:// www.nice.org.uk/guidance/CG127. NICE Guidance 2011. Accessed January 25, 2015.

7. Mancia G, Fagard R, Narkiewicz K, Redon J, Zanchetti A, Böhm M, et al. 2013 ESH/ESC Guidelines for the management of arterial hypertension. Eur Heart J 2013;34,2159-2219.

8. Hodgkinson J, Sheppard JP, Heneghan C, Martin U, Mant J, Roberts N, et al . Accuracy of ambulatory blood pressure monitors: a systematic review of validation studies. J Hypertens 2013; $31: 239-250$.

9. Sierra C. Associations between ambulatory blood pressure parameters and cerebral white matter lesions. Int J Hypertens 2011;2011:478710. 
10. PATS Collaborating Group. Post-stroke antihypertensive treatment study: a preliminary result. Chin Med J (Engl) 1995;108: 710-717.

11. PROGRESS Collaborative Study Subgroup. Randomized trial of perindopril based blood pressure-lowering regimen among 6108 individuals with previous stroke or transient ischemic attack. Lancet 2001;358:1033-1041.

12. Schrader J, Luders S, Kulschewski A, Hammersen F, Plate K, Berger J, et al. MOSES Study Group. Morbidity and Mortality After Stroke, Eprosartan Compared with Nitrendipine for Secondary Prevention: principal results of a prospective randomized controlled study (MOSES). Stroke 2005;36:1218-1226.

13. Yusuf S, Diener HC, Sacco RL, Cotton D, Ounpuu S, Lawton WA, et al. PRoFESS Study Group. Telmisartan to prevent recurrent stroke and cardiovascular events. N Engl J Med 2008; 359:1225-1227.

14. Benavente OR, Coffey CS, Conwit R, Hart RG, McClure LA, Pearce LA, et al. SPS3 Study Group. Blood-pressure targets in patients with recent lacunar stroke: the SPS3 randomised trial. Lancet 2013;382:507-515.

15. Jain S, Namboodri KKN, Kumari S, Prabhakar S. Loss of circadian rhythm of blood pressure following acute stroke. BMC Neurology 2004;4:1.

16. Castilla-Guerra L, Fernández-Moreno Mdel C, Espino-Montoro A, López-Chozas JM. Ambulatory blood pressure monitoring in stroke survivors: do we really control our patients? Eur J Intern Med 2009;20:760-763.

17. Sasaki M, Ando H, Fujimura A. Lack of nocturnal blood pressure fall in elderly bedridden hypertensive patients with cerebrovascular disease. Chronobiol Int 2012;29:62-65.

18. Cugini $P$, Tubani L, Sesana G, Capodaglio PF, Laganà B, Salandri $\mathrm{A}$, et al. The role and chronobiometric study of ambulatory arterial pressure monitoring in hypertensive patients with a recent cerebral ischemia episode. Recenti Prog Med 1997;88: 383-387.

19. Zakopoulos N, Spengos K, Tsivgoulis G, Zis V, Manios E, Vemmos K. Assessment of blood pressure control in hypertensive stroke survivors: an ambulatory blood pressure monitoring study. Blood Press Monit 2006; 11:235-241.

20. Kernan WN, Ovbiagele B, Black HR, Bravata DM, Chimowitz MI, Ezekowitz MD, et al. Guidelines for the prevention of stroke in patients with stroke and transient ischemic attack: a guideline for healthcare professionals from the American Heart Association/American Stroke Association. Stroke 2014;45: 2160-2236.

21. Castilla-Guerra L, Fernandez-Moreno Mdel C, Alvarez-Suero J, Jimenez-Hernandez MD. Can the treatment of arterial hypertension help to prevent dementia? Rev Neurol 2013;56:91-100.

22. Irie K, Yamaguchi T, Minematsu K, Omae T. The J-curve phenomenon in stroke recurrence. Stroke 1993;24:1844-1849.

23. Ovbiagele B, Diener HC, Yusuf S, Martin RH, Cotton D, Vinisko R, et al. PROFESS Investigators. Level of systolic blood pressure within the normal range and risk of recurrent stroke. JAMA 2011;306:2137-2144.

24. Ovbiagele B. Low-Normal systolic blood pressure and secondary stroke risk. J Stroke Cerebrovasc Dis 2013;22:633-638.

25. Castilla-Guerra L, Fernández-Moreno Mdel C, Espino-Montoro A, López-Chozas JM. Nocturnal hypotension in patients after ischemic stroke: does it really matter? Eur J Intern Med 2011;22:501-504.

26. Sare GM, Gray LJ, Wardlaw J, Chen C. ENOS Trial Investigators. Is lowering blood pressure hazardous in patients with significant ipsilateral carotid stenosis and acute ischaemic stroke? Interim assessment in the 'efficacy of nitric oxide in stroke' trial. Blood Press Monit 2009; 14:20-25.

27. Yamamoto Y, Akiguchi I, Oiwa K, Hayashi M, Kimura J. Adverse effect of nighttime blood pressure on the outcome of lacunar infarct patients. Stroke 1998;29:570-576.

28. Yamamoto Y, Akiguchi I, Oiwa K, Hayashi M, Kasai T, Ozasa K. Twenty-four-hour blood pressure and MRI as predictive factors for different outcomes in patients with lacunar infarct. Stroke 2002;33:297-305.

29. Staals J, van Oostenbrugge RJ, Knottnerus IL, Rouhl RP, Henskens LH, Lodder J. Brain microbleeds relate to higher ambulatory blood pressure levels in first-ever lacunar stroke patients. Stroke 2009;40:3264-3268.

30. Yamamoto Y, Ohara T, Nagakane Y, Tanaka E, Morii F, Koizumi T, et al. Chronic kidney disease, 24-h blood pressure and small vessel diseases are independently associated with cognitive impairment in lacunar infarct patients. Hypertens Res 2011;34:1276-1282. 